\title{
Silicon photonics for neuromorphic information processing
}

\author{
Peter Bienstman ${ }^{\mathrm{a}}$, Joni Dambre ${ }^{\mathrm{b}}$, Andrew Katumba ${ }^{\mathrm{a}}$, Matthias Freiberger ${ }^{\mathrm{b}}$, Floris Laporte $^{\mathrm{a}}$, \\ and Alessio Lugnan ${ }^{\mathrm{a}}$ \\ aPhotonics Research Group, Department of Information Technology, Ghent University - imec, \\ Belgium \\ ${ }^{\mathrm{b}}$ Data Science Lab, Department of Electronics and Information Systems, Ghent University - \\ imec, Belgium
}

\begin{abstract}
We present our latest results on silicon photonics neuromorphic information processing based a.o. on techniques like reservoir computing. We will discuss aspects like scalability, novel architectures for enhanced power efficiency, as well as all-optical readout. Additionally, we will touch upon new machine learning techniques to operate these integrated readouts. Finally, we will show how these systems can be used for high-speed low-power information processing for applications like recognition of biological cells.
\end{abstract}

Keywords: photonic neuromorphic information processing, reservoir computing

\section{INTRODUCTION}

The persistent increase in demand for systems that can process the massive amounts of data available today has strained the currently employed transistor-based von Neumann architectures. Simultaneously, the growing demand for high-throughput, high-fidelity telecommunications systems has generated significant implementation hurdles for the associated signal processing systems.

To address the compounding challenges for these computation and communication systems, a major design revolution is underway for the next generations of these systems in the IT research world. The frantic search for potential solutions has initiated a revisit to analog computation platforms but with the aim of combining them with the state-of-the-art in large-scale integration technology. These platforms exploit the inherent dynamics of certain physical systems for processing and/or computing. Of these, prominently under consideration are biologically inspired techniques, and particularly brain-inspired computing approaches that employ artificial structures that mimic the brain's neural computational semantics.

Reservoir computing $(\mathrm{RC})$ is a brain-inspired computing approach that initially emerged as a way around the intricacies associated with correctly training recurrent neural networks. ${ }^{1-3}$ Classical software RC involves setting up a large randomly initialized nonlinear dynamical system (the reservoir) - usually an artificial neural network - that is tuned into a specific dynamical regime to allow for the following three conditions: separability of the inputs, generation of similar outputs for similar inputs and some form of finite memory of the previous inputs. Under these circumstances, the states of the reservoir can be linearly combined, following task-imposed optimization criteria, to extract the desired outputs for the specified inputs.

Beyond the initial software implementations, RC has evolved into a way to enable computing with physical nonlinear dynamical systems. Examples of the concept applied to mechanical systems, memristive systems, atomic switch networks, boolean logic elements and photonic systems can be found in. ${ }^{4-8}$ Photonic RC particularly presents a number of benefits compared to e.g. electronics, as it offers a large bandwidth and is inherently massively parallel.

To date, experimental demonstrations of photonic reservoirs routinely achieve state of the art performance on various information processing tasks. Implementations based on a single nonlinear node with a delayed feedback architecture have proven that photonic RC is competitive for analog information processing. ${ }^{9-17}$ Moreover,

Corresponding author: Peter.Bienstman@UGent.be 
integrated photonic reservoirs can push computation speeds even higher for digital information processing. The performance of integrated photonic reservoirs has been studied numerically for networks of ring resonators, ${ }^{18-22}$ networks of SOAs, ${ }^{7}$ and experimentally with networks of delay lines and splitters in. ${ }^{23}$ Integrated photonic reservoirs are particularly compelling, especially when implemented in the CMOS platform as they can take advantage of its associated benefits for technology reuse and mass production.

A recent development in the design of RC systems is the realization that for certain tasks that are not strongly nonlinear, it is possible to achieve state-of-the-art performance using a completely passive linear network, i.e., one without amplification or nonlinear elements. The required nonlinearity is introduced at the readout point, typically with a photodetector. ${ }^{23}$ The work discussed in this paper is also based on this architecture. Aside from the integrated implementation introduced in, ${ }^{23}$ the passive architecture has been adapted to the single node with delayed feedback architecture in form of a coherently driven passive cavity. ${ }^{9}$

The rest of this paper is organised as follows. First, we discuss how passive reservoirs can benefit from improved power efficiency by distributing the same total input power over multiple inputs. Second, we introduce a novel hardware-friendly training scheme for devices with integrated all-optical readout. Finally, we present a spatial analog of reservoir computing which can be used to identify different types of biological cells in a flow-cytometry context.

\section{PASSIVE INTEGRATED PHOTONIC RESERVOIR COMPUTING}

We compare the performance of an architecture with the same size as in, ${ }^{23}$ with the same total input power injected into the reservoir but distributed over different nodes. We will show that even when the same power is injected into the reservoir, the increased variation between the reservoir states contributes considerably to the computing power of the architecture.

In this work, each considered combination of reservoir initialization and input configuration was tasked to solve the delayed XOR task. The current output bit for this task is the XOR of the current input bit with one $n_{\text {delay }}$ bits in the past. Here we express it as:

$$
y[n]=x[n] \oplus x\left[n-n_{\text {delay }}\right],
$$

where $x[n]$ is the bit-level representation of the input data stream and $y[n]$ is the bit-level representation of the output. Before injection into the reservoir, the inputs $(x[n])$ are converted from logical levels to discrete sampled data by upsampling and pulse shaping steps.

This task was considered as it is the most difficult of all delayed binary tasks involving only two bits. This is the case because, in machine learning terms, XOR is not linearly separable (see for example ${ }^{24}$ ).

For all considered input cases, the $4 \times 4$ (16 node) reservoir architecture was used to generate the states. This number of nodes was chosen as it is a design that is both cost-effective to produce with multi-project wafer runs, but also has a good performance on a number of tasks. In all cases, the length of the interconnections between the reservoir translates to a propagation time of $62.5 \mathrm{ps}$, matching the current generation of available chips.

For a fair comparison between the different cases, the same aggregate input power across all input nodes was used: $100 \mathrm{~mW}$. Where the input was fed into more than one node, the power was equally divided between the nodes. Results are reported as averages across 30 different random initialisations of the input weights and reservoir waveguide phases (each using different randomly generated bit streams).

Figure 1 shows averaged error rates plotted against total input power.

We observe that as we increase the number of the input nodes, the minimum power requirements for errorfree performance also go down. The most significant jump in power efficiency is an approximately 2 orders of magnitude decrease for the best 4-input node combination as compared to the 1 or 2 node input combinations. This can be attributed to the fact that the $[5,6,9,10]$ combination is the central loop in the swirl architecture which allows for significant signal distribution for a small number of inputs. We also observe that increasing the number of input nodes beyond 4 does not significantly impact the power efficiency. Since each input that needs to be driven incurs an additional hardware cost, we can conclude that driving the central four nodes is the most cost- and power-efficient solution. 

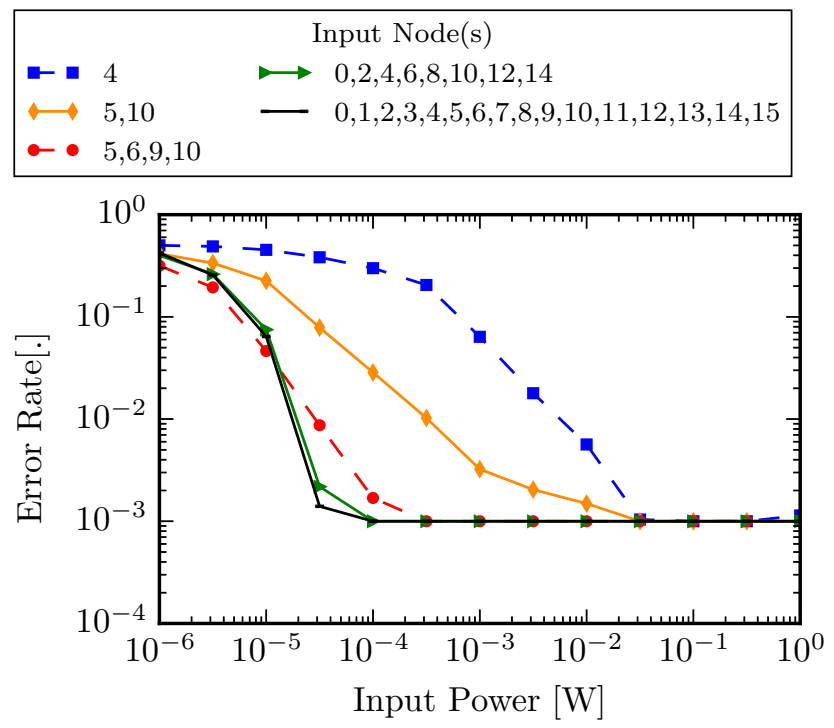

Figure 1: Error rate vs total input power for different injection scenarios. The minimum measurable error, given the number of bits used for testing, is $10^{-3}$.

\section{HARDWARE-FRIENDLY TRAINING ALGORITHMS}

Regular reservoir training algorithms, e.g., ridge regression or recursive least squares, assume full observability of all states of the reservoir. For a passive photonic reservoir with on-chip readout, this full observability is only obtained if a high-speed photodetector is implemented on the chip for each reservoir state to be read out. This multiple-detector approach is costly in terms of chip footprint and power consumption, and therefore we want to investigate an approach where the linear combination is happening in the optical domain and the result is being sent to a single photodiode. Since this loses full observability of the states, novel algorithms to train passive photonic reservoirs need to be found.

In order to achieve this, we exploit the weighting mechanism of the optical readout of the reservoir to read out all reservoir states through the single photodetector available at the end of the summing structure. Reading out the state variable $s_{i}$ in response to the training input sequence can be simply realized by setting the weight of that state variable to 1 and all other weights to 0 . By presenting the whole training input sequence to the reservoir $n$ times, where $n$ is the number of nodes of the reservoir, the training responses of all nodes can be collected through the single photodetector. By taking the square root of each measured power value, we can approximately invert the nonlinearity of the photodetector and obtain an estimate for the evolution of the light intensity over time at the corresponding reservoir node. However, since passive photonic reservoirs work with coherent light, it is not sufficient to know only the light intensities at the points predefined as reservoir states: we also need to know the corresponding phase of the light. While the absolute phase of the optical signal inside the reservoir is lost within the photodetection process, the relative phases between the optical state signals influence the power at the detector output. We therefore estimate the phase between two given optical signals within the integrated reservoir by obtaining the evolution of the sum of their states through time as we apply the training signal at the reservoir's input. We now use the phase of one state signal (node) as a reference. Using the evolution of the power of sum between the reference node's signal and each other node signal, as well as the previously determined powers of all individual states, we are able to estimate the relative phase of each node signal with respect to the reference node using basic trigonometric relationships. The last stage of this calculation consists of an inverse cosine, which is is injective, in the sense that there are always two solutions within the range $[-\pi, \pi]$. To discriminate between them, we perform a third measurement between the reference node's signal and each other node's signal, now shifting the phase of the reference node's readout weight by $\frac{\pi}{2}$ and comparing with the phase estimate obtained before. As a result, the whole process requires that we feed the training sequence through the reservoir $3 n-2$ times. Under ideal conditions, this nonlinearity inversion procedure 
is exact. Our simulations confirm that, when exchanging the true reservoir states in our setup with the states estimated through the method elaborated above, the resulting bit error plot is identical. Since detector noise is neglected in our current simple model of an integrated optical readout, future work will focus on extending the proof-of-concept nonlinearity inversion approach elaborated here to a more general setting incorporating a more realistic photodetector model.

\section{PILLAR SCATTERERS TO IMPROVE BIOLOGICAL CELL IDENTIFICATION}

The sorting of biological cells is of key importance in several biomedical applications, like diagnostics, therapeutics and cell biology. However, an accurate classification and separation of different cell types is usually expensive, time consuming and often requires alterations of the samples due to the use of labels, e.g. fluorescent tags, that may hinder subsequent analyses. ${ }^{25}$ For these reasons, the development of label-free, high-speed, automated and integrated cell sorting solutions is of particular interest. Among several options, the employment of digital holographic microscopy in microfluidic flow cytometry is a promising candidate. In this technique, the classification is carried out through the analysis of the interference pattern (hologram) projected by the cell when illuminated by monochromatic light. The hologram is acquired by an image sensor and contains information on the 3D refractive index structure of the cells. ${ }^{26}$ The large amount of information contained in a cell hologram enables nontrivial analysis and classifications. On the other hand, the computational cost of elaborating such a complex source of information by reconstructing the image from the hologram is a major hindrance to an increase in the cell sorter throughput, e.g. by parallelization of the process.

In this work, a passive, linear, integrated photonic stage is proposed as an interface between the hologram projection and the image sensor, in order to simplify the classification process in the relatively slow electric domain. In particular, the forward scattered light coming from a cell and the corresponding background are made to propagate through a collection of silica pillar scatterers of elliptic cross section embedded in silicon nitride (Fig. 2). For computational time reasons, this process has been investigated via 2D finite-difference timedomain simulations as a proof-of-concept, approximating the 3D case of a cell flowing in a microfluidic channel interfaced with a photonic chip. The far-field intensity of the light exiting the scatterers cluster is collected by an array of virtual pixels that approximately simulate a line scan image sensor. The pixel outputs are fed into a linear classifier that can be, for example, implemented in the electric domain.

The photonic stage containing the scatterers is intended to exploit the nonlinearity of the transfer function that relates the phase shift accumulated by the light through the cell to the corresponding interference pattern measured by an image sensor.

Let us consider a green laser source $(\lambda=532 \mathrm{~nm})$ and let us compare the classification error on the test samples when no scatterers are present and when, instead, 4 scatterer layers are employed (considering the random displacement amplitude $A_{r}=150 \mathrm{~nm}$ and the layer distance $D=1.846 \mu \mathrm{m}$ ). Let us stress that the error rate expected value and the confidence intervals drawn in all the following graphs are extracted from the results obtained from 20 random permutations of the simulated samples and correspond to an optimal choice of the number of training epochs. The resulting error rate values for different numbers of pixels and for different noise levels (Fig. 3) show that the use of scatterer layers allows for a significant error rate reduction (up to $\sim 50 \%$ ), provided that a sufficient number of pixels and a low enough noise level are considered. The increased sensitivity of classification performance towards added noise level when scatterers are used is ascribed to the fact that the scatterers presence unfolds the cell diffraction pattern into a higher number of components that may be important for classification. Thus, it is probable that some of these components have low intensity with respect to the average pattern intensity and are therefore easily overcome by high relative levels of noise.

The chosen scatterer configuration was the best performing among the tested combinations (800 simulated samples each). Performances similar to the ones presented in this paper were obtained for a broad number of cases. Therefore, the main conclusion of this (non-exhaustive) geometrical exploration is that the employment of scatterer layers of different kinds can decrease the classification error rate significantly, up to $50 \%$. 



Figure 2: Schematic of the classification process. From right to left: a monochromatic plane wave impinges on a microfluidic channel containing a cell in water $\left(n_{\mathrm{H}_{2} \mathrm{O}} \sim 1.34\right)$, which has a low refractive index contrast $\left(n_{\text {cytoplasm }}=1.37, n_{\text {nucleus }}=1.39\right)$; the forward scattered light passes through a collection of silica scatterers $\left(n_{\mathrm{SiO}_{2}} \sim 1.461\right)$ embedded in silicon nitride $\left(n_{\mathrm{Si}_{3} \mathrm{~N}_{4}} \sim 2.027\right)$ and organized in layers; the radiation intensity is then collected by a far-field monitor, which is divided into bins (pixels); each pixel value is fed into a trained logistic regression, which classifies the cell as a "normal" cell (small nucleus) or as a "cancer" cell (big nucleus). The logistic regression consists of a weighted sum of the pixel values. The weights are trained so that the sum exceeds a threshold value only if a certain input class is recognized.

\section{CONCLUSIONS}

We have presented a study showing that distributing the available input power of different input channels is beneficial for performance and scalability. Moreover, we presented a hardware-friendly training mechanism that deals with the limited observability in case the linear combination is performed entirely in the optical domain. Finally, we introduced a spatial analog of reservoir computing - an array of pillar scatterers - which can boost the performance of cell-sorting applications.

\section{Acknowledgments}

This research was funded by the EU Horizon 2020 PHRESCO Grant (Grant No 688579), the BELSPO IAP P7-35 program Photonics@be and the Research Foundation Flanders (FWO)(Grant No G024715N).

\section{REFERENCES}

[1] Maass, W., Natschläger, T., and Markram, H., "Real-time computing without stable states: A new framework for neural computation based on perturbations," Neural computation 2560, 2531-2560 (2002).

[2] Jaeger, H. and Haas, H., "Harnessing nonlinearity: predicting chaotic systems and saving energy in wireless communication.," Science (New York, N.Y.) 304, 78-80 (2004).

[3] Verstraeten, D., Schrauwen, B., D'Haene, M., and Stroobandt, D., "An experimental unification of reservoir computing methods," Neural Networks 20, 391-403 (4 2007).

[4] Hauser, H., Ijspeert, A., Füchslin, R., Pfeifer, R., and Maass, W., "Towards a theoretical foundation for morphological computation with compliant bodies," Biological Cybernetics 105, 355-370 (12 2011).

[5] Sillin, H. O., Aguilera, R., Shieh, H.-H., Avizienis, A. V., Aono, M., Stieg, A. Z., and Gimzewski, J. K., "A theoretical and experimental study of neuromorphic atomic switch networks for reservoir computing.," Nanotechnology 24, 384004 (2013). 
a)

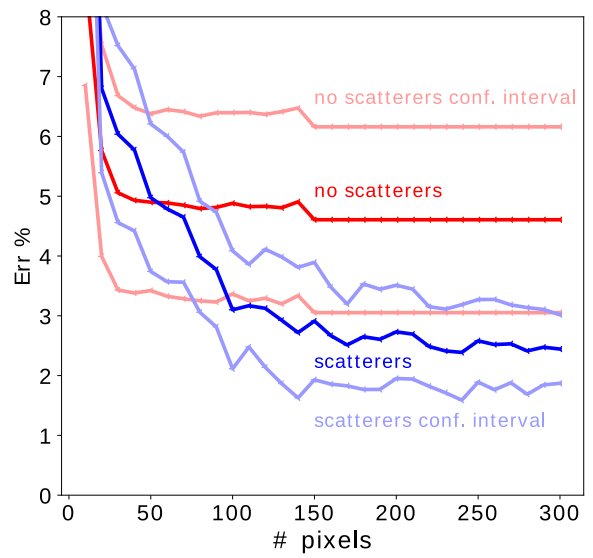

b)

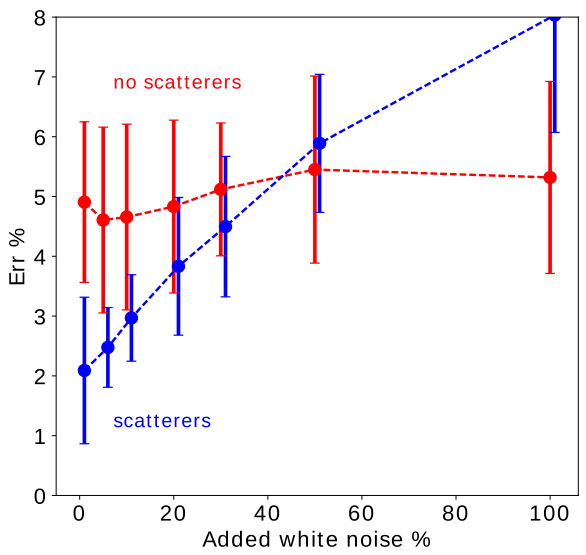

Figure 3: Comparison between the test error rates of "normal" and "cancer" cell classification, corresponding to the absence (in red) and the presence (in blue) of scatterers). A green laser source $(\lambda=532 \mathrm{~nm})$ is employed. a) Test error rate as a function of the number of employed pixels, with $5 \%$ added white noise. The darker and the lighter versions of the two line colors respectively represent the mean value and the confidence interval (of 2 standard deviations) over the 20 sample sets generated for validation. b) Test error rate (averaged on the values obtained considering $N_{\text {pix }}=250,260, \ldots, 300$ ) as a function of the added noise percentage. In order to avoid error bar overlap, some of the blue points are slightly shifted to the right. Both the plots show that the scatterers' presence allows for an error rate reduction up to $\sim 50 \%$, provided that a sufficient number of pixels and a low enough noise level are considered.

[6] Kulkarni, M. S. and Teuscher, C., "Memristor-based reservoir computing," in [Proceedings of the 2012 IEEE/ACM International Symposium on Nanoscale Architectures - NANOARCH '12], 226-232, ACM Press, New York, New York, USA (2012).

[7] Vandoorne, K., Photonic reservoir computing with a network of coupled semiconductor optical amplifiers, $\mathrm{PhD}$ thesis (2011).

[8] Paquot, Y., Duport, F., Smerieri, A., Dambre, J., Schrauwen, B., Haelterman, M., and Massar, S., "Optoelectronic Reservoir Computing," Scientific Reports 2, 287 (2 2012).

[9] Vinckier, Q., Duport, F., Smerieri, A., Vandoorne, K., Bienstman, P., Haelterman, M., and Massar, S., "High-performance photonic reservoir computer based on a coherently driven passive cavity," Optica 2(5), 438-446 (2015).

[10] Brunner, D., Soriano, M. C., Mirasso, C. R., and Fischer, I., "Parallel photonic information processing at gigabyte per second data rates using transient states.," Nature communications 4, 1364 (1 2013).

[11] Appeltant, L., Soriano, M. C., Van der Sande, G., Danckaert, J., Massar, S., Dambre, J., Schrauwen, B., Mirasso, C. R., and Fischer, I., "Information processing using a single dynamical node as complex system.," Nature communications 2, 468 (9 2011).

[12] Larger, L., Soriano, M. C., Brunner, D., Appeltant, L., Gutierrez, J. M., Pesquera, L., Mirasso, C. R., and Fischer, I., "Photonic information processing beyond Turing: an optoelectronic implementation of reservoir computing," Optics Express 20, 3241 (1 2012).

[13] Duport, F., Schneider, B., Smerieri, A., Haelterman, M., and Massar, S., "All-optical reservoir computing," Optics Express 20, 22783 (9 2012).

[14] Dejonckheere, A., Duport, F., Smerieri, A., Fang, L., Oudar, J.-L., Haelterman, M., and Massar, S., "Alloptical reservoir computer based on saturation of absorption," Optics Express 22, 10868 (5 2014).

[15] Soriano, M. C., Ortín, S., Brunner, D., Larger, L., Mirasso, C. R., Fischer, I., and Pesquera, L., "Optoelectronic reservoir computing: tackling noise-induced performance degradation," Optics Express 21, 12 (1 2013). 
[16] Nguimdo, R. M., Verschaffelt, G., Danckaert, J., and Van der Sande, G., "Fast photonic information processing using semiconductor lasers with delayed optical feedback: Role of phase dynamics," Optics Express 22, 8672 (4 2014).

[17] Hicke, K., Escalona-Morán, M., Brunner, D., Soriano, M. C., Fischer, I., and Mirasso, C. R., "Information Processing Using Transient Dynamics of Semiconductor Lasers Subject to Delayed Feedback," IEEE Journal of Selected Topics in Quantum Electronics 19, 1501610-1501610 (7 2013).

[18] Vandoorne, K., Dambre, J., Verstraeten, D., Schrauwen, B., and Bienstman, P., "Parallel reservoir computing using optical amplifiers.," IEEE transactions on neural networks 22, 1469-81 (9 2011).

[19] Mesaritakis, C., Papataxiarhis, V., and Syvridis, D., "Micro ring resonators as building blocks for an alloptical high-speed reservoir-computing bit-pattern-recognition system," JOSA B (October) (2013).

[20] Fiers, M. A. A., Van Vaerenbergh, T., Wyffels, F., Verstraeten, D., Schrauwen, B., Dambre, J., and Bienstman, P., "Nanophotonic reservoir computing with photonic crystal cavities to generate periodic patterns," IEEE Transactions on Neural Networks and Learning Systems 25(2), 344-355 (2014).

[21] Zhang, H., Feng, X., Li, B., Wang, Y., Cui, K., Liu, F., and Dou, W., "Integrated photonic reservoir computing based on hierarchical time-multiplexing structure," Opt. Express 22, 31356-31370 (12 2014).

[22] Mesaritakis, C., Kapsalis, A., and Syvridis, D., "All-Optical Reservoir Computing system based on InGaAsP Ring Resonators for High-Speed Identification and Optical Routing in Optical Networks," 9370, 1-7 (2015).

[23] Vandoorne, K., Mechet, P., Van Vaerenbergh, T., Fiers, M., Morthier, G., Verstraeten, D., Schrauwen, B., Dambre, J., and Bienstman, P., "Experimental demonstration of reservoir computing on a silicon photonics chip.," Nature communications 5, 3541 (1 2014).

[24] Theodoridis, S. and Koutroumbas, K., "04 Nonlinear Classifiers," Pattern Recognition (Fourth Edition), 151-260 (2009).

[25] Gossett, D. R., Weaver, W. M., Mach, A. J., Hur, S. C., Tse, H. T. K., Lee, W., Amini, H., and Di Carlo, D., "Label-free cell separation and sorting in microfluidic systems," ANALYTICAL AND BIOANALYTICAL CHEMISTRY 397, 3249-3267 (AUG 2010).

[26] Lagae, L., Vercruysse, D., Dusa, A., Liu, C., de Wijs, K., Stahl, R., Vanmeerbeeck, G., Majeed, B., Li, Y., and Peumans, P., "High throughput cell sorter based on lensfree imaging of cells," in [2015 IEEE INTERNATIONAL ELECTRON DEVICES MEETING (IEDM)], IEEE, 345 E 47TH ST, NEW YORK, NY 10017 USA (2015). IEEE International Electron Devices Meeting (IEDM), Washington, DC, DEC 07-09, 2015. 